\title{
Low Frequency Vibration of Multiwall Carbon Nanotubes with Heterogeneous Boundaries
}

\author{
R. Chowdhury, C.Y. Wang*, and S. Adhikari \\ School of Engineering, Swansea University \\ Singleton Park, Swansea, Wales SA2 8PP, UK
}

Key words: multiwall carbon nanotubes, vibration, heterogeneous end constraint

\begin{abstract}
Heterogeneous end constraints are imposed on multiwall carbon nanotubes (MWCNTs) by sequentially clamping one end of their originally simply supported constituent tubes. The finite element method is employed to study the vibration of such MWCNTs with an emphasis on the effect of the mixed boundary conditions. The results show that the clamping process constantly enhances the dynamic stiffness of MWCNTs, which leads to substantial frequency increase up to $50 \%$ and in some cases, the transformation of the fundamental vibration mode. In particular, the vibration frequency is always found to be most sensitive to fixing the outermost tubes, showing the critical role of this individual tube in determining the structural stiffness of the whole MWCNTs as a coupled system.
\end{abstract}

\footnotetext{
* Corresponding author: Chengyuan.wang@swansea.ac.uk
} 


\section{Introduction}

Carbon nanotubes (CNTs) [1] with extreme stiffness and low mass density [2-3] show great potential to serve as nanoresonators in nanoelectronics, nanodevices and nanoelectromechanical systems [4, 6-9]. The vibration of CNTs is thus crucial for their successful applications in nanotechnology. Specifically, some vibration modes of CNTs, e.g., radial breathing mode [10-13], beam-like bending mode [5, 14] and longitudinal mode [15], offers valuable probes for the molecular structures and the elastic properties of CNTs. On the other hand, CNTs consisting of straight concentric layers with circular cross-section could lose their structural symmetry due to the vibration in axial, circumferential and radial directions [1617]. This could result in a sudden change in their physical properties (e.g., the electrical properties [18]) and in turn, significantly affect their performance in nanostructures. Thus, similar to the buckling behaviour [19] the vibration of CNTs turns out to be a major topic of great interest in nanomechanics. In the last two decades, considerable efforts [20] have been devoted to capturing the fundamental vibration behaviours of CNTs by using experimental techniques [10-11, 14-15] and multi-scale modelling tools [12-13, 16-17, 21-23].

Recently, the interest of the mechanics of CNTs has been transferred from their fundamental behaviours to the effect of internal and external factors on the elastic properties [2426], buckling [27-30] and vibration [31] of CNTs. Such factors include thermal effects [25, 3234], the defects of CNTs [25-27, 32], the initial stresses [31] and the constraints on the two ends of CNTs [29-30, 31]. Interesting phenomena have been observed, such as the negative Poisson ratio of defective single wall CNTs (SWCNTs) [26] and the variation of buckling modes due to the switch of the end constraints on multiwall CNTs (MWCNTs) [30]. 
The end constraint on CNTs has been identified as a major external factor that exerts strong effects on the buckling of CNTs [29, 30]. This issue is of practical interest because surrounding media will inevitably enforce a variety of constraints on the two ends of CNTs. In particular, different constraints could be imposed on the individual tubes of MWCNTs, i.e., the heterogeneous end constraints of MWCNTs. As a typical example, while the outer tube of a double wall CNT is clamped the inner tube may still be free or simply supported. To our best knowledge, such heterogeneous constraints were not considered for MWCNTs until our recent buckling analysis of MWCNTs [30]. Specifically, the vibration analysis of the MWCNTs with mixed boundary conditions still remains absent in the literature. It is thus of great interest to further study the vibration of the MWCNTs with heterogeneous end constraints.

The present paper is focused on the effect of the heterogeneous end constraints on the low frequency vibration modes of MWCNTs, which are of major engineering interest. The mixed end constraints will be achieved by sequentially clamping one end of the constituent tubes of originally simply supported MWCNTs. This task is challenging for analytic methods and formidable for atomistic simulations. The finite element method will thus be employed in the present analysis. Three types of MWCNTs will be considered, such as thin and short, thick and short, and (almost) solid and slender MWCNTs. Thin, thick and (almost) solid MWCNTs are defined by the innermost radius-to-thickness ratio $>5, \sim 1$ and $<0.25$, and short and slender ones are defined by the length-to-the outermost diameter aspect ratio $\sim 5$ and $\sim 10$. The vibration modes will be captured and their frequency dependence on the number of clamped tubes will be calculated for the three types of MWCNTs studied here. 


\section{Finite element method for vibration of MWCNTs}

MWCNTs comprise two to dozens layers of coaxial constituent SWCNTs. The individual tubes are coupled via the normal interlayer van der Waals (vdW) interaction. The friction between neighbouring tubes is usually very low and thus, will be neglected in the present study. The model used in the present work for the free vibration analysis of MWCNTs are described in this section.

In the present study, each constituent tube of MWCNTs will be modelled as an elastic shell with equivalent wall thickness $h$, Young's modulus $E$ and Poisson ratio $v$. When deformation occurs, the radial pressure between adjacent tubes due to the interlayer vdW interaction is calculated by $p=c \cdot\left(w_{0}-w_{i}\right)$ and the interaction between the other (non-adjacent) tubes as well as the interlayer friction is ignored. Here, $w_{0}$ and $w_{i}$ denote the radial displacements of the outer and inner tubes of two adjacent layers, respectively. The constant $c$ is the vdW interaction coefficient defined by the second derivative of the interlayer potential energy with respect to the interlayer spacing. The value of $c$ at the equilibrium interlayer spacing $0.34 \mathrm{~nm}$ is estimated as $c=102 \mathrm{GPa} / \mathrm{nm}[13,35]$. Here, the analytic method formulated for the MWCNTs with homogeneous end constraints [13,17,35-37] cannot be further used in the present study where heterogeneous end constraints are imposed. To circumvent this problem, a finite element (FE) technique has been developed based on the methodology suggested by Wang et. al [29]. The solid shell element is adopted, and the mesh design is selected such that each tube of MWCNTs consists of 36 elements in the cross sectional area and $\frac{45 L}{10 R_{i}}$ elements in the longitudinal direction. Here, $R_{i}$ is the innermost radius and $L$ is the length of MWCNTs. The radial force between adjacent tubes due to the interlayer vdW interaction is calculated based on a linear 
spring model where the spring constant $K=\frac{c}{2}\left(A_{i}+A_{o}\right)$ is obtained by multiplying the pressure relationship by equivalent area $\frac{A_{i}+A_{o}}{2}$, where $A_{i}$ and $A_{o}$ are the mid-areas of the inner and outer elements, respectively [30]. Obviously, the stiffness $K$ is a constant for a specific tube but varies between different individual tubes. For more details regarding the FE modelling technique, reader may refer to Ref. [29]. Here, the ANSYS FE software package is used to perform the buckling analysis of MWCNTs. In particular, this ANSYS model has been efficiently used in the buckling analysis of MWCNTs with various homogeneous and heterogeneous boundary conditions [30], where a good agreement has been achieved between the present ANSYS model and other FE modes and analytical methods.

In the present vibration analysis of MWCNTs, the ends of each constituent SWCNT are subjected to one of the following sets of boundary conditions, i.e.,

(1) The simply-supported (SS) boundary condition: $u=0, v=0$ and $w=0$, and

(2) The clamped (C) boundary conditions: $u=0, v=0, w=0$ and $\frac{\partial w}{\partial x}=\frac{\partial w}{\partial \theta}=0$

Here $u, v$ and $w$ are the displacements of the individual tubes in longitudinal, circumferential and radial directions, respectively. In the FE analysis, imposing the clamped end constraints on CNTs is straight forward and can be done by constraining the nodes of the CNT end(s) for displacements and rotations. The simply-supported ends of solid cylinders can also be achieved simply by restricting the tangential displacement of the concentric end nodes while allowing their rotation about any axes. This method, however, is not applicable for hollow CNTs where nodes do not exist in the concentric axis. To solve this problem, we shall introduce an imaginary plane at the end of CNTs, which encloses the hollow CNTs and enables the aforementioned constraint to be applied to its concentric nodes, thus reflecting a simply-supported end of CNTs. 
Here it should be emphasized that the imaginary plane is not involved in the vibration of MWCNTs since the constrained are applied on that plane. For this reason it will not have significant effect on the vibrational modes and associated frequencies.

\section{Low Frequency Vibration of MWCNTs}

In what follows, we shall use the above obtained ANSYS model to study the five low frequency vibrations of MWCNTs with heterogeneous end constraints, i.e., constituent tubes of the MWCNTs have different boundary conditions. To introduce the heterogeneity at boundaries we sequentially clamp one end of the constituent tubes of originally simply supported MWCNTs until all the constituent tubes are consistently clamped at one end while the other end is still simply supported. This clamping process is carried out in the following sequences: clamp the outermost tube first, then its adjacent tube... and finally the innermost tube. This procedure yields various heterogeneous boundary conditions where increasing number of the layers are clamped at one end and simply supported at the other, while other layers are simply supported at both ends. The present study will thus be focused on the effect of such boundary conditions on the low frequency vibrations of MWCNTs. In particular, five-wall CNTs will be used as a typical example of MWCNTs, which are classified into three categories as shown in Table 1, i.e., (1) thin and short (Example 1), (2) thick and short (Example 2) and (3) almost solid and slender

(Example 3) MWCNTs where $\frac{R_{i}}{H}=5,1$ and 0.25, and $\frac{L}{D_{0}}=5,5$ and 10, respectively. Here, $H$ denotes the thickness of MWCNTs and $D_{0}$ is the outermost diameter of the MWCNTs. The values of equivalent material and geometric constants of constituent SWCNTs used in the 
present study are Young's modulus $E=3.5 T P a$, the effective wall thickness $H=0.1 \mathrm{~nm}$ and Poisson ratio $v=0.2[38,39]$. The vibration modes of the lowest five frequencies are captured for the three examples and the associated frequencies are calculated as functions of the number $(N)$, i.e., the number of the layers with one end clamped and the other simply supported. The results are shown in Figs. 1, 2 and 3 for examples 1, 2 and 3, respectively.

\subsection{Thin and short MWCNTs}

Let us first study the vibration of example 1, a thin and short five-wall CNT with $R_{i} / H=5$ and $L / D_{0}=5$. In this example, the five constituent SWCNTs have a radius rising from $5 \mathrm{~nm}$ to $6.36 \mathrm{~nm}$, and the length-to-diameter aspect ratio between 5 and 6.3. Obviously, they are shell-type constituent tubes with comparable radius and the aspect ratio. In Fig. 1a, the thin five-wall CNT is observed to behave like a single-layer elastic thin shell where the five constituent tubes vibrate collectively with nearly identical displacements. As a result, in Fig. 1 a, there is no significant change in the interlayer spacing during the vibrations. This unique feature remains unchanged no matter what mixed boundary condition is imposed. Here the vibration modes can be characterised approximately by $(n, m)$, where $n$ is the circumferential wave number and $m$ is the half axial wave number. Since example 1 has large radial size and thus, a low radial rigidity, the circumferential wave number $n$ of modes I, II, III, IV and V increases from 2 to 3 and to 4 . In the mean time, the value of $m$ only varies between 1 and 2 .

Fig.1b shows that, in general, the frequency of the five vibration modes in Fig. 1a increases monotonically with the increase of the number $(N)$ of the tubes with one end clamped. This is simply because enforcing constraints on the rotation of the tube ends can normally strengthen the constituent tube and thus, improve the dynamic stiffness of the whole MWCNT. This effect of 
the clamped ends is found to be most significant for mode I associated with the lowest frequency. It then becomes less pronounced for modes III and V associated with higher frequencies. Indeed, as seen from Fig. 1b, when the number $N$ increases from zero to five, the frequency of mode I with $(n, m)=(2,1)$ increases from $18 G H z$ to $25 G H z$. The frequency shift is $7 \mathrm{GHz}$ and the relative change defined by the frequency shift-to-the initial frequency $(18 \mathrm{GHz})$ ratio is $39 \%$. In the same process, the frequency of mode III with $(n, m)=(3,2)$, rises by $5.1 \mathrm{GHz}$ from its initial value $39.7 \mathrm{GHz}$ giving a relative change $12.85 \%$ and that of mode $\mathrm{V}$ with $(n, m)=$ $(4,2)$ increases by $1.5 \mathrm{GHz}$ from $55.4 \mathrm{GHz}$ corresponding to a relative change $2.7 \%$. It is well known that the vibration displacement amplitude of shell-structures usually decreases with increasing vibration frequency. Thus, clamping the ends of shell structures is expected to exert stronger influence on the lower frequency vibration with larger displacement amplitudes. This offers a plausible explanation for the decreasing effect of the boundary condition on the vibration modes with higher frequencies. Next, let us further study mode II and IV of example 1 (Fig. 1a). It is seen from Fig. $1 \mathrm{~b}$ that with $N$ rising from 0 to 5 , the frequency shift of mode II and IV is $1.4 \mathrm{GHz}$ and $0.4 \mathrm{GHz}$ corresponding to the relative change $5 \%$ and $0.77 \%$, respectively. Thus, as compared with modes III and V, modes II and IV are less sensitive to the change in the end constraint in spite of the fact that their frequencies are lower than those of mode III and mode V, respectively. Here it is seen from Fig.1 a that the axial half wave number of modes II and IV is $m$ $=1$ while that of modes III and V is $m=2$, i.e., the axial wavelength of modes III and V are half of those of modes II and IV. Accordingly, for simply supported example 1, the end rotary angle in axial direction of modes III and V could be significantly larger than those of modes II and IV. In view of this analysis, it follows that imposing restriction on the end rotation in axial direction (i.e., $\frac{\partial w}{\partial x}=0$ ) tends to exert more significant impacts on the vibration with larger axial half wave 
number $m$ or a smaller axial wavelength. In other words, raising the axial half wave number tends to enhance the effect of clamped ends on the vibration of CNTs. This can at least partially explain the aforementioned comparison between modes III and V, and modes II and IV.

MWCNTs are a coupled system comprising SWCNTs of different radii and the aspect ratios. Thus, the contribution of the individual tubes (i.e., SWCNTs) to the structural stiffness of MWCNTs could be significantly different. To exam this issue, we have calculated the frequency shift due to the rotation restriction on one single layer based on the data shown in Fig. 1b. Here let us label the outermost layer tube 1 , the next layer tube $2, \ldots$ and the innermost layer tube 5 and denote the frequency up-shift obtained by fixing one end of tube $1,2, \ldots$, and 5 as $\Delta f_{1}, \Delta f_{2}$ $\Delta f_{3} \Delta f_{4}$ and $\Delta f_{5}$, respectively. The frequency up-shift ratio $\frac{\Delta f_{k}}{\Delta f_{5}}$ is plotted for all the five modes of example 1 in Fig. 4a. Here $k=1,2 \ldots 5$ is the number of each constituent tube. It is interesting to note in Fig. 4a that, for the five vibration modes of thin example 1 the frequency up-shift ratio $\frac{\Delta f_{k}}{\Delta f_{5}}$ decreases monotonically when the tube number $k$ increases from 1 to 5 . In particular, $\Delta f_{1}$ due to clamping the outermost layer is around 5 to 15 times of $\Delta f_{5}$ achieved by fixing the innermost tube only. These results clearly show that, among the constituent tubes of example 1, the outmost one contributes greatly to the dynamic structural stiffness of the MWCNT. The contribution of the constituent tubes then declines rapidly from the outermost tube to the innermost one. For examples 2 and 3, similar calculation has been done and the results are shown graphically in Fig. 4b and c, respectively, which will be discussed in details in the following sections. 


\subsection{Thick and short MWCNTs}

Example 2 is a thick and short five-wall CNT with $R_{i} / H=1$ and $L / D_{0}=5$ (see Table 1 ) which is a mixture of four shell-type tubes with the aspect ratio 5 to 8 , i.e., the outer four SWCNTs, and one beam-type tube with the aspect ratio 10, i.e., the innermost SWCNT. The five vibration modes of this example are shown in Fig. 2a where modes I, III, IV and V are the shelllike vibrations similar to those observed in Fig. 1a while mode II is a beam-like vibration which is not seen in Fig.1a. The shell-like vibration of example 2 can be attributed to the four shell-type constituent tubes in the five-wall CNT, while the beam-like vibration is a result of the core beam-type tube and the coupling between adjacent tubes via the interlayer vdW interaction. Here, contrary to the case of thin example 1, the radii of individual tubes differ considerably in thick example 2, which, in turn, results in noticeable difference in their transverse displacements and the significant changes in the interlayer spacing (see the cross-sections in Fig. 2a). It is easy to understand that thick example 2 with the innermost radius $1 \mathrm{~nm}$ displays higher radial stiffness than that of thin example 1 with the innermost radius $5 \mathrm{~nm}$. Thus, when the vibration mode changes from modes I to $\mathrm{V}$, the circumferential wave number $n$ of example 2 alters between 1 and 3 while the axial half wave number $m$ increases from 1 to 4 (Fig. 2a). This behaviour is different from that of thin example 1 , where from modes I to $\mathrm{V}, n$ rises substantially from 1 to 4 but $m$ only varies between 1 and 2 (Fig. 1a).

Fig. $2 \mathrm{~b}$ shows the number $N$-dependence of the frequencies associated with the five vibration modes illustrated in Fig. 2a. It is seen from Fig. $2 b$ that all the frequencies tend to increase with the increasing number $N$. Specifically, clamping one end of all the five layers raises the frequency of model I by $48 \mathrm{GHz}$. Considering its initial frequency (at $N=0) 116.3 \mathrm{GHz}$ the relative increment is calculated as $41.2 \%$. For modes III $(m=2)$ and V $(m=4)$, respectively, 
with higher frequencies, the corresponding frequency shift decreases to $23.3 \mathrm{GHz}$ and $30.2 \mathrm{GHz}$, and the relative increase reduces to $10.9 \%$ and $5.6 \%$, which, however, are still larger than the frequency shift $7.06 \mathrm{GHz}$ and $9.1 \mathrm{GHz}$, and the relative increase $4.65 \%$ and $2 \%$ obtained for modes II $(m=1)$ and IV $(m=3)$, respectively. These behaviours are qualitatively similar to those observed for example 1 and thus, can be understood based on the same theories. In addition, it is noted that the relative frequency changes of mode I to mode $\mathrm{V}$ obtained for example 2 are $41.2 \%, 4.7 \%, 11 \%, 2 \%$ and $5.6 \%$, respectively, close to those of example 1, i.e., $39 \%, 5 \%, 12 \%$, $0.77 \%$ and $2.7 \%$. However, since the frequencies (or the dynamic stiffness) of example 2 are around 10 times as much as their counterparts of thin example 1 the absolute frequency shift of example 2 are 6 to 20 times that of thin example 1. Furthermore, Fig. $4 \mathrm{~b}$ shows that for the five modes of example 2, the frequency up-shift obtained by clamping one single layer decreases from the outermost tube to the innermost one with the value of $\frac{\Delta f_{1}}{\Delta f_{5}}$ around 9 to 20 for modes I to IV. It is noted that the slopes of the curves in Fig. $4 \mathrm{~b}$ are generally greater than those shown in Fig. 4a for example 1, which means that the outermost few tubes of example 2 generally play an more important role in supporting the whole MWCNT than those of thin example 1. This can be explained by the fact that, for thick example 2 substantial difference in the radius and the aspect ratio exists between the constituent tubes, whereas such difference becomes much smaller in thin example 1.

As shown above, clamping the constituent tubes has stronger impacts on mode I than mode II. In Fig. 2 b, when $N=0$, i.e., the simply supported boundary condition for example 2, the frequency of mode I is $34.6 \mathrm{GHz}$ lower than that of model II; it then catches up with the frequency of model II when $N$ rises to 3, i.e., one end of the outermost three layers is clamped. Further fixing the inner tube or the innermost two tubes the frequency of mode I turns out to be 
even higher than that of mode II. In other words, for example 2, the vibration mode associated with the fundamental frequency will transform from shell-like vibration with $m=1$ and $n=2$ to beam-like vibration with $m=n=1$ when the one end of the tubes is clamped consecutively. Here, it is reasonable to expect that such a mode transformation would occur earlier, i.e., at smaller $N$, and result in the model I frequency much higher that of mode II, when both ends of the constituent tubes are clamped. In practical applicaitons, the fundamental mode of shell structures is of major interest, and the alteration of this vibration could either significantly affect the performance of CNT-based nanostructures or have some potential applications directly exploiting this unique feature. The output of the present study can thus provide useful guidance to facilitate the design and development of these CNT-based nanotechnology. Here it is worth mentioning that, similar transformation due to the boundary condition change has also been observed for the critical buckling mode of the thick and short MWCNTs [30].

\subsection{Solid and slender MWCNTs}

In this section, we shall focus on a (almost) solid and slender five-wall CNT, i.e., example 3 in Table 1. It is easy to see that this example consists of five beam-type SWCNTs whose aspect ratio increases from 10 (the outermost tube) to 50 (the innermost tube), much larger than those of examples 1 and 2. On the other hand, example 3 is (almost) solid with a small innermost radius $0.34 \mathrm{~nm}$. It therefore exhibits radial stiffness even higher than that of example 2. As a result, it is seen in Fig. 3a that, in modes I, II, III and IV, the vibration of example 3 follows the beam-like bending modes where $n=1$ remains unchanged (i.e., the cross-section is circular without any deformation) while $m$ increases substantially from 1 to 4 . In clamping one end of the constituent 
tubes, the maximum frequency shift obtained for modes I, II, III and IV is $24.8 \mathrm{GHz}, 37 \mathrm{GHz}$, $41.3 \mathrm{GHz}$ and $43.7 \mathrm{GHz}$, respectively, (Fig. 3b) and the corresponding relative change is calculated as $52 \%, 20.6 \%, 11.3 \%$ and $7.6 \%$. These values are significantly larger than those obtained for shell-like vibration of examples 1 and 2, showing that the beam modes of a slender CNT are more sensitive to clamping the constituent tubes in axial direction. This observation can probably be explained by the fact that the energy of the beam modes of slender example 3 is completely determined by its bending in axial direction. However, the energy of the shell-like vibrations of short examples 1 and 2 comes not only from off-plane bending but also from inplane stretching/compression of the tube walls. On the other hand, it is interesting to see in Fig. $2 \mathrm{~b}$ that, in contrast to the case of slender example 3 , the beam mode of short example 2 (mode II in Fig. 2a) cannot be significantly affected by the clamped tube ends. Here the major difference is that, without the interlayer vdW interaction, the five beam-type tubes of example 3 would still vibrate in the same beam-like bending mode whereas, in the thick and short example 2, while its beam-type tube is likely to oscillate in the beam mode, other four shell-type tubes would be inclined to vibrate in shell-like modes. Thus, the predominant beam mode achieved in example 2 is originated from its beam-type innermost tube and the coupling between the motions of the neighbouring tubes. In other word, the interlayer vdW interaction plays a more significant role in the bending mode of example 2 (mode II). This naturally leads to less pronounced end constraint effect on the beam-like vibration of example 2. In addition, shell-like vibration is also observed for slender example 3 (mode $\mathrm{V}$ in Fig. 3a), whose frequency is found to be almost a constant independent of the number $N$ of clamped tubes (Fig. 3b). 
The frequency up-shift $\Delta f_{k}(k=1,2 \ldots 5)$ due to one clamped tube is also calculated for modes I to IV of example 3 . The ratio $\frac{\Delta f_{k}}{\Delta f_{5}}(k=1,2 \ldots .5)$ is presented in Fig. 4 c. As seen from the figure, the tendency of $\frac{\Delta f_{k}}{\Delta f_{5}}(k=1,2 \ldots 5)$ to change with the tube number $k$ is similar to those shown in Fig. $4 \mathrm{a}$ and $\mathrm{b}$ for examples 1 and 2. On the other hand, $\frac{\Delta f_{k}}{\Delta f_{5}}(k=1,2 \ldots 5)$ of example 1 increases greatly from modes I to mode $\mathrm{V}$, and is up to more than one order of magnitude larger than those of examples 1 and 2 (Fig. $4 \mathrm{a}$ and b). This reveals that clamping one end of the outer tubes (with a larger radius) has much stronger effect on the structural stiffness of slender MWCNTs vibrating in beam modes. Specifically, this effect turns out to be more prominent for the beam like vibrations with a larger axial half wave number or a smaller half wavelength. The more important role of the outer tubes in example 3 can be attributed to the fact that the dynamic stiffness of the MWCNT vibrating in beam modes is largely dependent on the inertial moment of the constituent tubes, which increases almost exponentially from the innermost tube to the outermost one. Further, the strain and stress withstood by individual tubes also rise linearly with the radius of these tubes which are bent like elastic beams.

\section{CONCLUSIONS}

The finite element method has been used to study the low frequency vibration of MWCNTs with heterogeneous end constraints, which cannot be easily conducted by using analytical methods. The heterogeneity is introduced by sequentially clamping one end of the constituent tubes of originally simply supported MWCNTs. The attention of this study is thus 
focused on the effect of clamping the tube ends on the free vibration of MWCNTs. Three types of MWCNTs are considered, namely, thin and short, thick and short, and (almost) solid and slender MWCNTs defined by $R_{i} / H \geq 5, \approx 1$ and $\leq 0.25$, and $L / D_{0} \leq 5$ and $\geq 10$, respectively. The major conclusions arising from the present study are summarized as follows,

1. Clamping the ends of constituent tubes of MWCNTs significantly enhances the dynamic stiffness of the whole MWCNTs. Thus, sequentially fixing the ends of these tubes results in continuous frequency up-shift for almost all vibration modes of three types of MWCNTs under consideration.

2. The most significant effect of clamping the tube ends occurs for the vibration mode I associated with the lowest frequency and normally, the largest displacement amplitude. Such an effect then decreases for the vibration modes II to $\mathrm{V}$ with higher frequencies and usually, smaller displacement amplitudes. The maximum frequency increase around 40 to $50 \%$ has been achieved for mode I and up to $20 \%$ for modes II to V of the three MWCNTs studied here.

3. In addition, enforcing clamped end on the outermost tube of MWCNTs can most efficiently upshift the frequency of all the vibration modes for the three types of MWCNTs. The result shows that the outmost tube plays the most important role in determining the dynamic stiffness of MWCNTs as a coupled system. The contribution of individual tubes to the structural stiffness of MWCNTs then declines from the outermost layer to the innermost one.

4. For a thick and short MWCNT, consecutively fixing its constituent tubes not only upshifts its vibration frequency but also transforms its fundamental vibration modes, e.g., from the shelllike vibration with deformation in both axial and circumferential directions to the beam-like 
vibration with bending in axial direction only. Such a mode transformation could have potential influences on the application and proper functioning of CNT-based nanostructures.

\section{REFERENCES}

[1] Iijima S 1991 Nature 56354

[2] Yakobson B I and Richard E S 1997 American Scientist 85, 324

[3] Qian D, Wagner J G, Liu W K, Yu M F and Ruoff R S 2002 Appl. Mech. Rev. 55, 495

[4] Treacy M M J, Ebbesen T W and Gibson J M 1996 Nature 381, 678

[5] Li C Y and Chou T W 2003 Phys Rev B 68, 073405

[6] Baughman R H, Zakhidov A A and de Heer W A 2002 Science 297, 787

[7] Lau K T and Hui D 2002 Composites B 33, 263

[8] Sazonova V, Yaish Y, Ustunel H, Roundy D, Arias T A and McEuen P L 2004 Nature 431, 284

[9] Kwon Y W, Manthena C, Oh J J and Srivasta D 2005, J. Nanosci. Nanotechnol. 5, 703

[10] Rao AM, Richter E, Bandow SJ, Chase B, Eklund PC, Williams K A, Fang S, Subbaswamy KR, Menon M, Thess A, Smalley R E, Dresselhaus G and Dresselhaus MS 1997 Science 275, 187

[11] Bandow S and Asaka S 1998 Phys. Rev. Lett. 80, 3779

[12] Popov VN and Henrard L 2002 Phys. Rev. B 65, 235415

[13] Wang CY, Ru CQ, Mioduchowski A 2005 J. Appl. Phys. 97, 024310

[14] Wang ZL, Poncharal W A and de Heer W A 2000 J. Phys. Chem. Solids 61, 1025 
[15] Bottani CE, Bassi AL, Beghi MG, Podesta A, Milani P, Zakhidov A, Baugham R, Walters DA and Smalley RE 2003 Phys. Rev. B 67, 155407

[16] Yoon J, Ru CQ and Mioduchowski A 2002 Phys. Rev. B 66, 233402

[17] Wang CY, Ru CQ, Mioduchowski A 2005 Phys. Rev. B 72, 075414

[18] Tang DS, Bao ZX, Wang LJ, Chen LC, Sun LF, Liu ZQ, Zhou WY and Xie SS 2000, J. Phys. Chem. Solid 61, 1175

[19] Wang CY, Zhang YY, Wang CM, and Tan, VBC, 2007 J. Nanosci. Nanotechno. 7, 4221

[20] Gibson RF, Avorinde EO and Wen YF 2007 Compos. Sci. Technol. 67

[21] Li CY and Chou TW 2003 Appl. Phys. Lett. 84, 121

[22] Wang CY, Ru CQ, Mioduchowski A 2005 J. Appl. Phys. 97, 114323

[23] Zhou J and Dong JM 2007 Appl. Phys. Lett. 91, 173108

[24] Xia YY, Zhao MW, Ma YC, Ying MJ, Liu XD, Liu PJ and Mei LM 2002 Phys. Rev. B 65,155415

[25] Jeng YR, Tsaia PC and Fang TH 2004 J. Phys. Chem. Solid 65, 1849

[26] Scarpa F, Adhikari S. and Wang CY 2009 J. Phys. D-Appl. Phys. 42, 142002.

[27] Shen HS and Zhang CL 2006 Phys. Rev. B 74035410

[28] Liew KM, Wang JB, He XQ and Zhang HW 2007 J. Appl. Phys. 102, 053511

[29] Wang CM, Ma Y Q, Zhang YY and Ang KK 2006 J. Appl. Phys. 99, 114317.

[30] Tong FM, Wang CY and Adhikari S 2009 J. Appl. Phys. 105, 094325

[31] Sun C and Liu K 2007 Solid State Comm. 143, 202

[32] She HS and Zhang CL 2006 Phys. Rev. B 74, 35410

[33] Yao X H and Han Q 2006 J. Eng. Master. Technol. 128, 419

[34] Zhang C L and Shen H S 2007 Phys. Rev. B 75, 45408 
[35] Wang CY and Mioduchowski A 2007 J. Appl. Phys. 101, 14306

[36] Wang CY, Ru CQ and Mioduchowski A 2003 J. Nansci. Nanotechnol. 3, 192

[37] Wang CY, Ru CQ and Mioduchowski A 2004 J. Appl. Mech. 71, 622

[38] Wang CY and Zhang L C 2008 Nanotechnology 19, 075705

[39] Wang CY and Zhang L C 2008 Nanotechnology 19, 195704 
Table 1 Five-wall CNTs as typical examples of MWCNTs considered in the present study

\begin{tabular}{cccc}
\hline & 1 & 2 & 3 \\
\cline { 2 - 4 } & Thin & Thick & Solid/Slender \\
\hline$R_{i}(\mathrm{~nm})$ & 6.8 & 1.36 & 0.34 \\
$H(\mathrm{~nm})$ & 1.36 & 1.36 & 1.36 \\
$L(\mathrm{~nm})$ & 81.6 & 27.2 & 34 \\
$L /\left(D_{0}\right)$ & 5 & 5 & 10 \\
$R_{i} / H$ & 5 & 1 & 0.25 \\
\hline
\end{tabular}




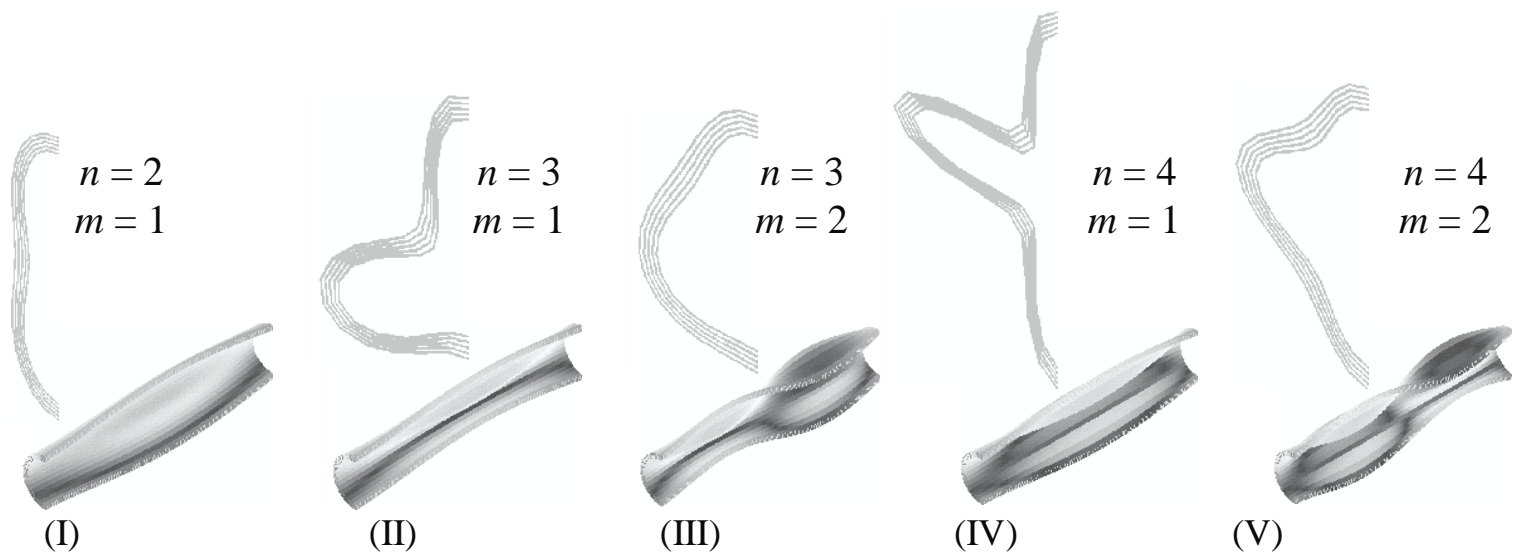

(a)

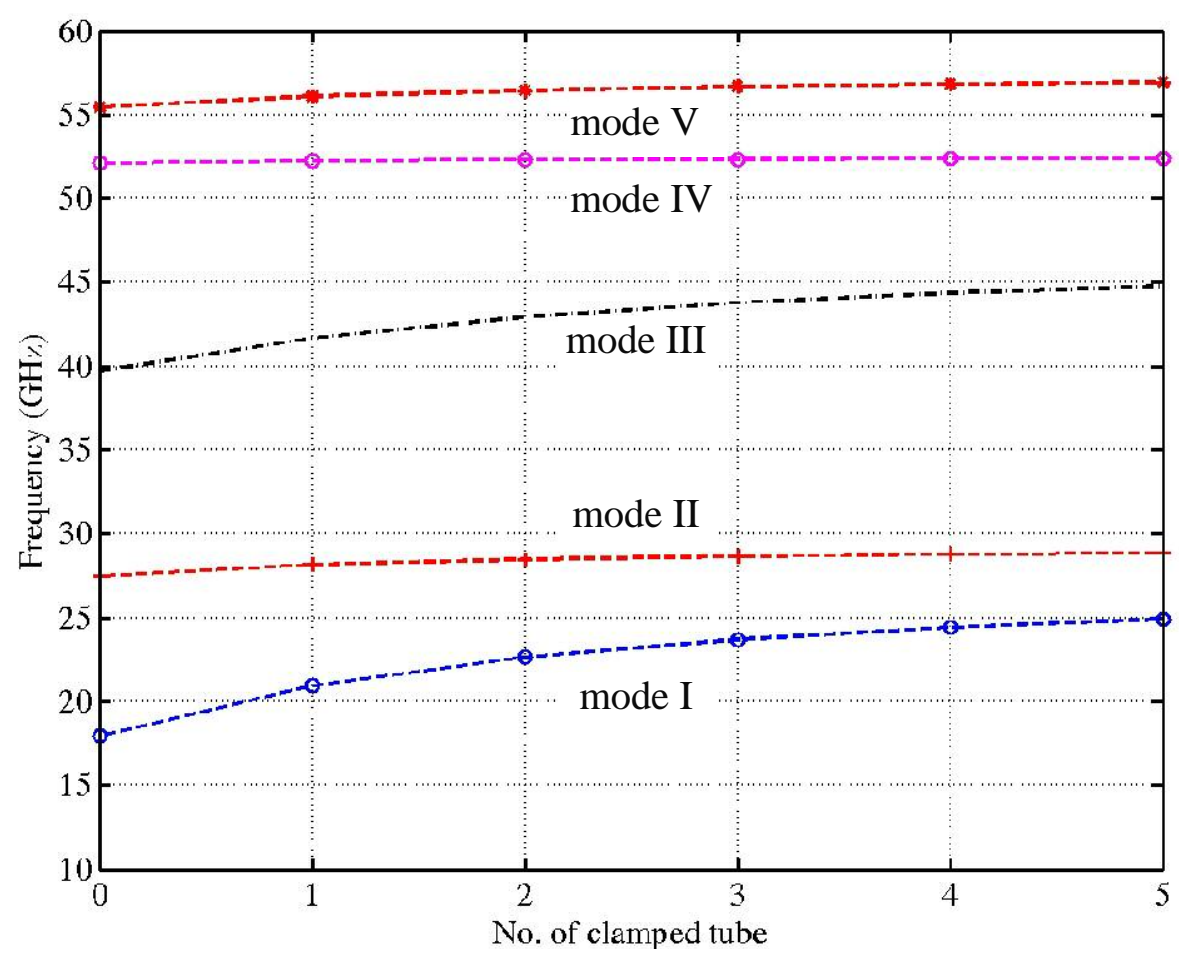

(b)

Fig.1 (a) The five low frequency vibration modes of Example 1, i.e., mode I, II, III, IV and V, and (b) the dependence of the frequency associated with the five modes on the number of tubes with one end clamped. 


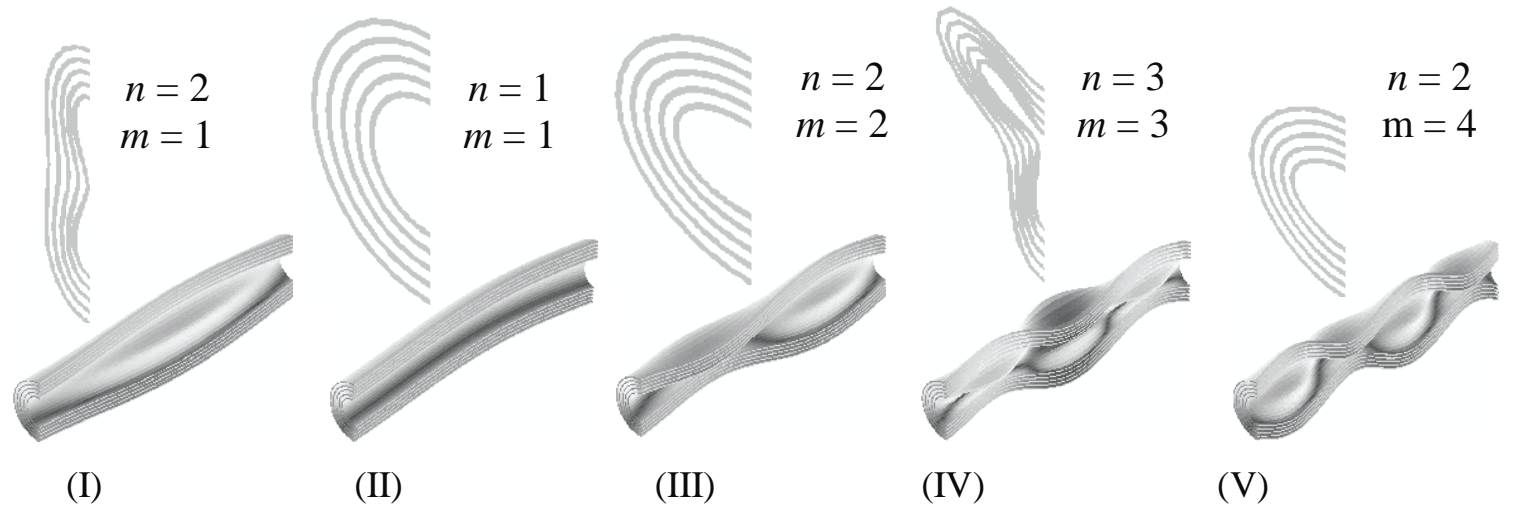

(a)

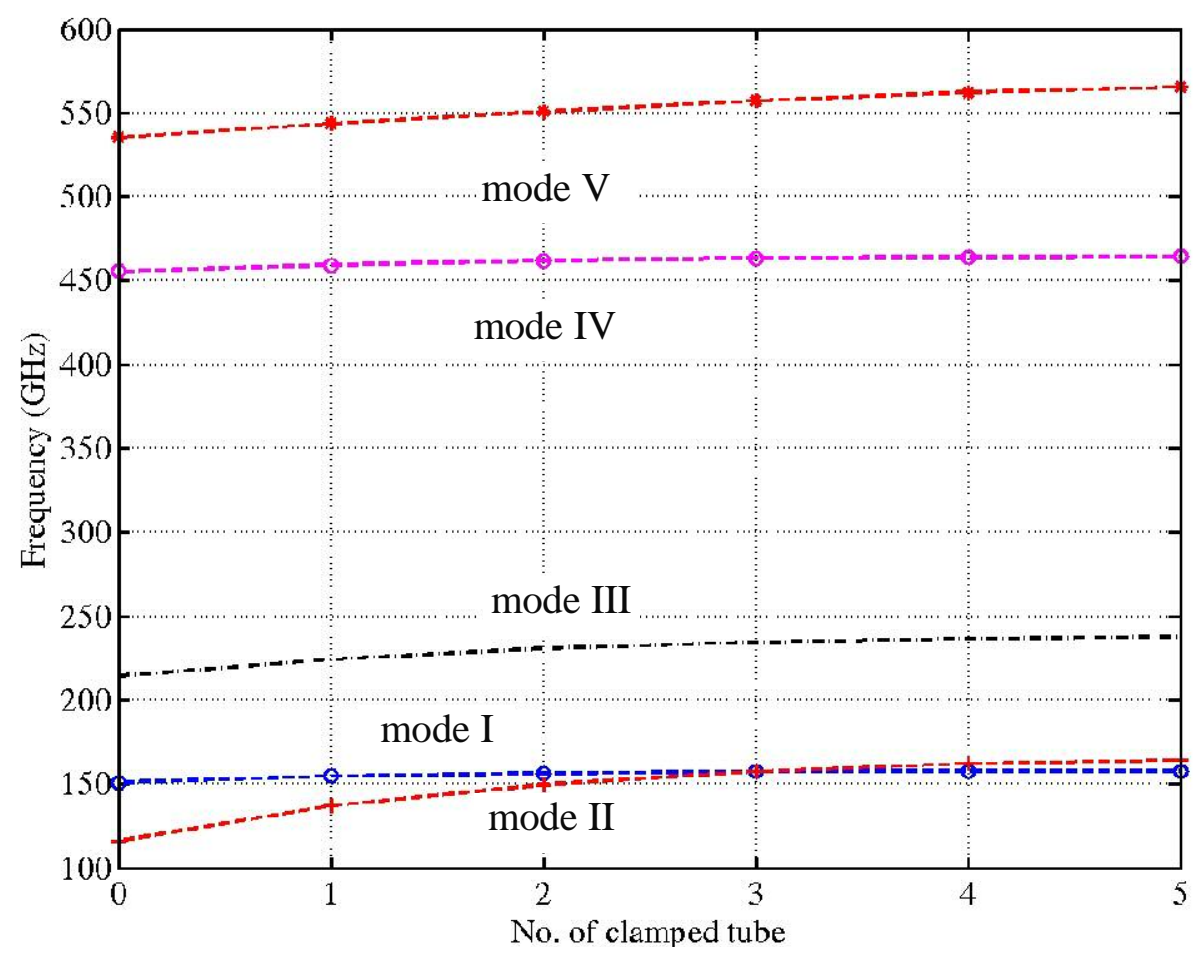

(b)

Fig.2 (a) The five low frequency vibration modes of Example 2, i.e., mode I, II, III, IV and V, and (b) the dependence of the frequency associated with the five modes on the number of tubes with one end clamped. 


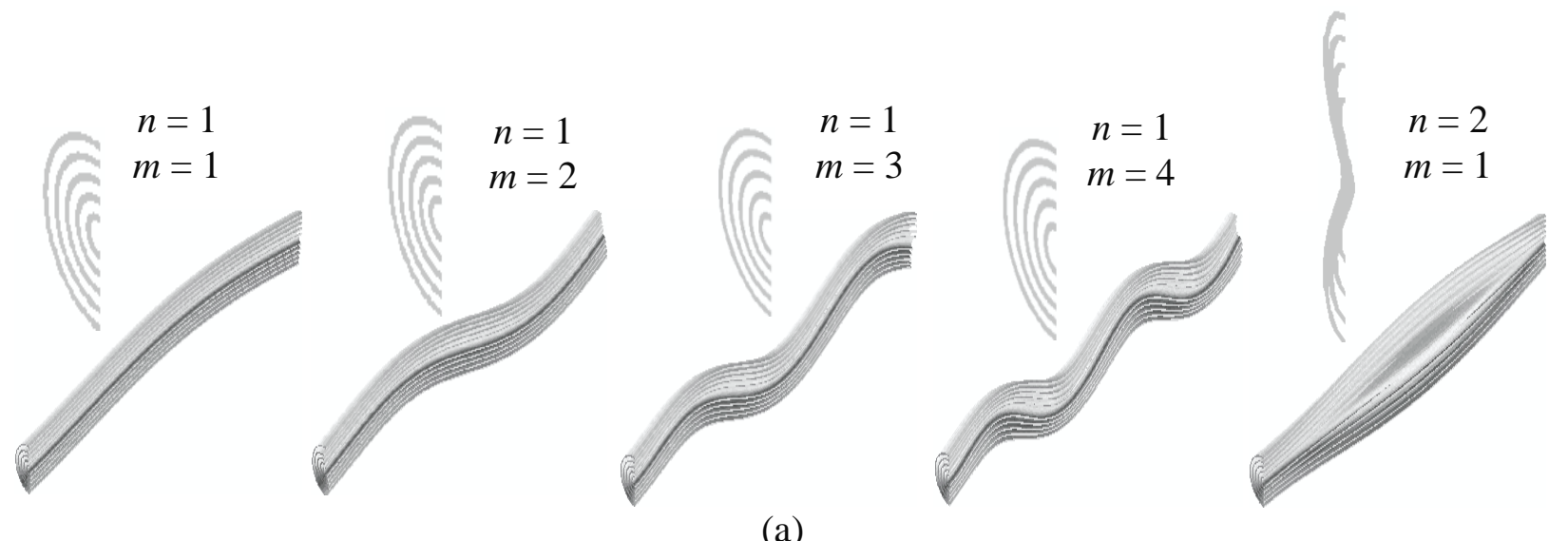

(a)

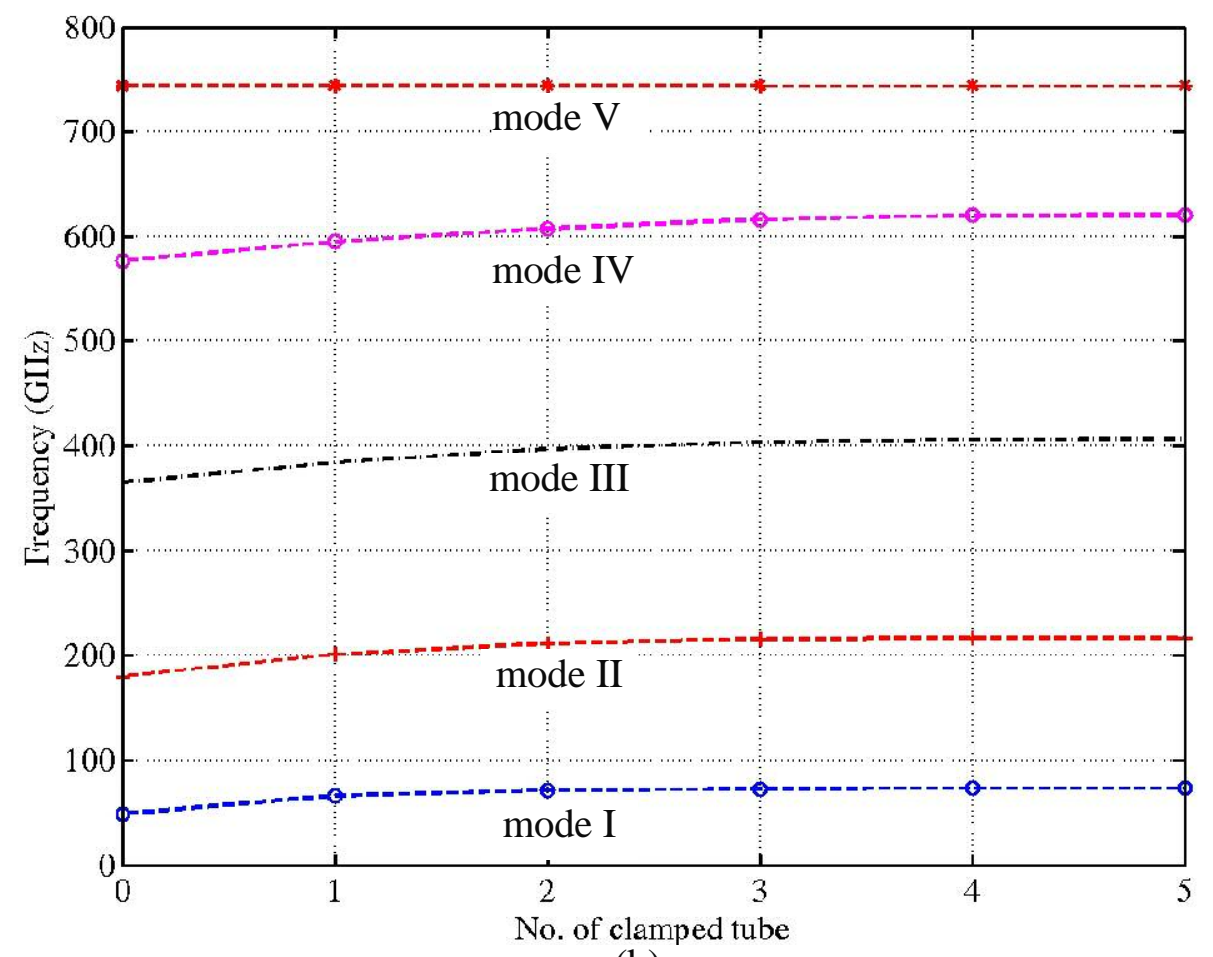

(b)

Fig.3 (a) The five low frequency vibration modes of Example 3, i.e., mode I, II, III, IV and V, and (b) the dependence of the frequency associated with the five modes on the number of tubes with one end clamped. 


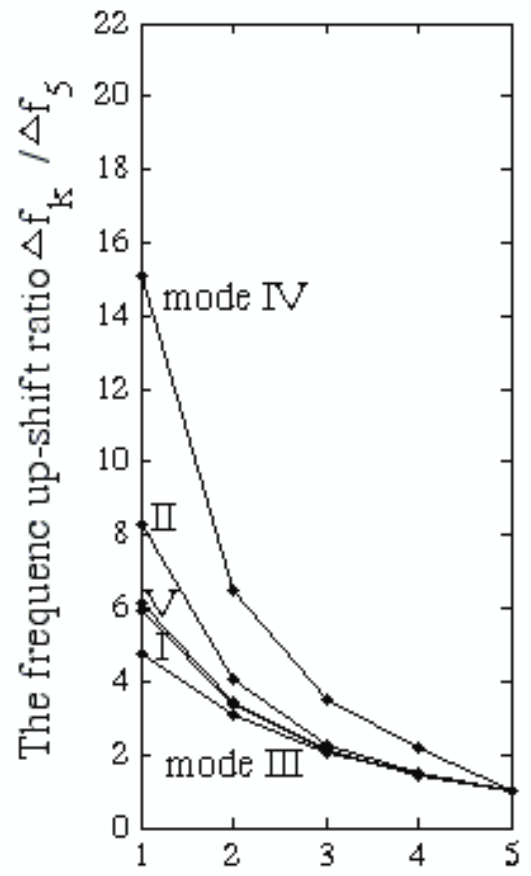

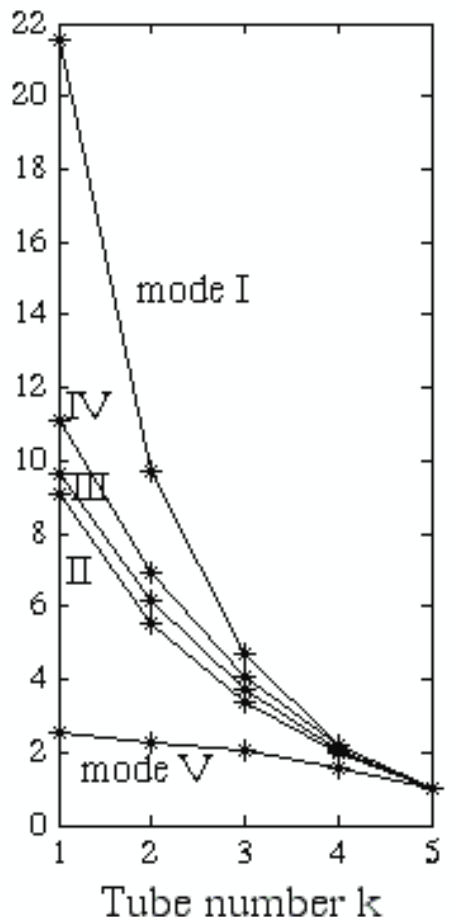

(b)

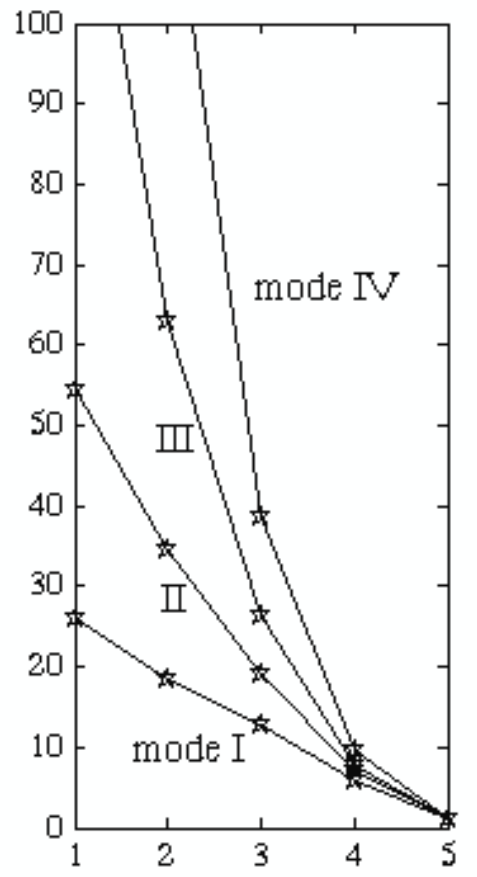

(c)

Fig. 4 The frequency up-shift ratio $\frac{\Delta f_{k}}{\Delta f_{5}}(k=1,2 \ldots 5)$ as a function of tube number $k(k=1$, 2...5) calculated for modes I, II, III, IV and V of (a) example 1, (b) example 2 and (c) example 3 in Table 1. Here $\Delta f_{1}, \Delta f_{2} \quad \Delta f_{3} \quad \Delta f_{4}$ and $\Delta f_{5}$ represent the frequency up-shift achieved by clamping the outmost tube 1 , the next tube $2 \ldots$ and finally, the innermost tube 5 in the process of sequentially clamping one end of constituent tubes described in section 3 . 